\title{
Two-hop time synchronization protocol for sensor networks
}

Jing Wang ${ }^{*}$, Shuai Zhang, Dan Gao and Yingguan Wang

\begin{abstract}
One of the critical middleware services for sensor networks is the time synchronization, which provides supports to different applications. Synchronization protocols used for Internet and LANs are not appropriate in the sensor networks for the high-density and limited energy resource. This paper describes two-hop time synchronization (TTS) that aims at reducing the synchronization overhead and providing more accurate network-wide synchronization. The synchronization message exchanges are minimized by making full use of sensors' broadcast domain and enlarging the common node synchronization range in multi-hop scenarios. By halving synchronization hops, the TTS achieves high multi-hop synchronization precision. The proposed protocol contains single-hop synchronization model, multi-hop synchronization algorithm, and a power control scheme. We prove that the extension of single-hop TTS to network-wide synchronization is NP-complete. The complexity and convergence time of multi-hop TTS are analyzed in detail. We simulate TTS on MATLAB and show that it requires minimal overhead and convergence time compared with other protocols. We also implement TTS on common sensors and its multi-hop synchronization error is less than that of receiver-receiver synchronization (R-RS).
\end{abstract}

Keywords: Sensor networks; Time synchronization; Synchronization hops; Synchronization range; Multi-hop

\section{Introduction}

The technological advances in miniaturization, in digital circuits design, and in wireless communication are promoting the study of sensor networks with small and low-cost nodes. By interacting with the environment and communicating with each other, the sensors could provide future ubiquitous communications. When those sensor nodes are deployed over a wide geographical region, they form the wireless sensor networks (WSN). They are useful for providing applications varying from environment monitoring to equipment monitoring, from smart office to industrial automation [1].

Clock synchronization is one of the most important components in WSN. It is essential for transmission scheduling, power management, data fusion, and many other applications. For example, in power management, the duty cycling helps the sensors to maintain energy by consuming minimal power during the sleep mode. The performance of duty cycling is closely related to the accuracy of the whole network synchronization.

\footnotetext{
*Correspondence: wangjingchn@163.com

Shanghai Institute of Microsystem And Information Technology, Chinese

Academy of Sciences, Shanghai 201899, China
}

Although the time synchronization problem has been solved in common networks, it requires to be reconsidered in WSN. First, such networks are energy constraint, so the time synchronization protocol must be energy efficient. Second, nodes in sensor networks communicate with each other via multi-hop paths. The single-hop error accumulates along the synchronization hops, and the multi-hop error is inevitable. Sometimes, the multi-hop synchronization error even becomes a major consideration due to the large network scale.

\subsection{Our contribution}

We propose the single-hop TTS to solve the disadvantages of traditional synchronization models. The model has the same time accuracy with receiver-receiver synchronization (R-RS), and its reference node can also be synchronized. Our main contribution is the multi-hop TTS protocol, which is the extension of single-hop TTS. We prove that this extension process is NP-complete and present a new distributed algorithm to accomplish it. The fundamental property of multi-hop TTS is that it synchronizes nodes within two adjacent levels in one synchronization process. In our design, reference node and its parent

\section{每 Springer}

(c) 2014 Wang et al.; licensee Springer. This is an Open Access article distributed under the terms of the Creative Commons Attribution License (http://creativecommons.org/licenses/by/2.0), which permits unrestricted use, distribution, and reproduction in any medium, provided the original work is properly cited. 
node perform two-way timing message exchange to synchronize nodes lying within the reference node broadcast domain. The TTS minimizes the reference nodes needed to cover the entire network and has the largest common node synchronization range in multi-hop scenarios. So it could reduce multi-hop synchronization overhead significantly. Our approach decreases multi-hop synchronization error by halving the synchronization hops. We analyze the complexity and convergence time of multihop TTS, and the result shows that our work is simple and fast. Odd layer TTS, which uses odd layer nodes as the reference nodes, is another achievement of this paper. This scheme is used to balance the energy consumption of nodes in different levels.

\section{Related work and challenges}

The most important single-hop synchronization models are receiver-receiver synchronization, pair-wise synchronization (P-WS) and sender-receiver synchronization (S-RS).

In R-RS, different nodes that receive the same reference message can be synchronized with each other by exchanging the recorded reception time. References $[2,3]$ and $[4]$ are based on reference broadcast synchronization (RBS) which is the most important prototype of R-RS. RBS achieves higher time accuracy compared with other protocols by removing the non-determinism of the sending end. However, in RBS, the reference node is left unsynchronized.

P-WS uses pair-wise message exchanges to achieve the synchronization of two adjacent nodes. Protocols that adopt this model are lightweight time synchronization (LTS) [5], tiny-sync and mini-sync [6], and timing-sync protocol for sensor networks (TPSN) [7].

S-RS synchronizes a receiver with a sender by broadcasting multiple time packets. The flooding time synchronization protocol (FTSP) [8] and delay measurement time synchronization (DMTS) [9] analyze the sources of uncertain delay in message exchange in detail. FTSP utilizes media access control (MAC) time stamping to minimize the clock offset. It also adopts linear regression to compensate the clock skew.

Recently, some new protocols have been proposed to solve the drawbacks of the above models. In pair-wise broadcast synchronization (PBS) [10], a node gets synchronized by overhearing the exchanged messages from two neighbors. In vector Kalman filter using multiple parents (KFMP) [11], a node combines the messages from multiple parents and adopts vector Kalman filter to reduce the global clock error. The master synchronization [12] adopts physical layer feedback control to save power consumption. Recently, a new approach called Average TimeSynch (ATS) [13] is proposed to estimate the clock offset and skew by utilizing distributed low-pass filter. Although some multi-hop protocols have been proposed to fit the above models, the multi-hop clock synchronization is still challenging for several reasons. On one hand, the synchronization hops become a major consideration for better clock protocols design. This is because the multi-hop synchronization error accumulates along the hops. For example, RBS proves that the multi-hop error increases with the square root of the hops. In order to reduce the cumulative multi-hop error, most algorithms focus on designing more accurate single-hop model or adopting a statistical signal processing framework. However, we find that all the current multi-hop protocols virtually share the same synchronization hop: the least hop to the root node. So our work aims at reducing the synchronization hops and providing precise multi-hop synchronization.

Synchronization overhead is another challenge that multi-hop synchronization faces due to the limited power sources. Actually, the overhead (energy consumption) is the opposite of time accuracy. The three basic models focus on improving the synchronization accuracy, so their synchronization overheads are usually very large. In [14] and [15], some multi-hop extensions of PBS have been devised. The extensions aim to minimize the number of synchronization packets. However, their effects are unsatisfactory because the synchronization range of PBS is too small. Synchronization range is the key factor for the energy-efficient protocol design. No protocol notices that, in multi-hop synchronization, the synchronization range of a common node is smaller than the single-hop synchronization range.

\section{The single-hop model of TTS}

The TTS is designed to achieve the synchronization of the whole network. In general, the communication radius of a sensor is assumed to be less than a few hundred meters. In this case, the propagation delay can be ignored. Since the energy consumption increases with the broadcast radius, this assumption is consistent with common sensor networks.

The single-hop TTS focuses on providing synchronization points (sync-points), which can be used to estimate the node clock phase and skew, instead of the approach to calculate the offsets directly.

In Figure 1a, suppose that $P$ is a parent node and $A$ is a reference node. Common node $B$, which lies within the broadcast region of $A$, collects sync-points by overhearing the information from $A$. The message exchange model is depicted in Figure 2.

1. A broadcasts $N$ reference packets and records the sending time $T_{i}^{A A}(1 \leq i \leq N)$. 


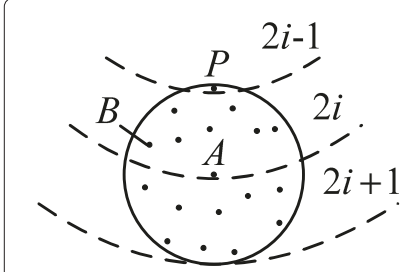

(a)

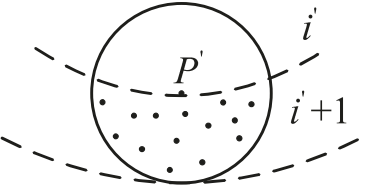

(b)
Figure 1 Synchronization examples of TTS and traditional protocols. (a) Synchronization example of TTS. (b) Synchronization example of traditional protocols.

2. Each receiver records the time stamp at reference message reception. The reception time of $P$ and $B$ are $T_{i}^{A P}$ and $T_{i}^{A B}$, respectively.

3. $P$ forwards a packet containing $N$ recorded time stamps to $A$.

4. A broadcasts the packet received from $P$.

From Figure 2, $T_{1}^{A P}$ and $T_{i}^{A P}$ can be expressed as

$$
\begin{aligned}
& T_{1}^{A P}=T_{1}^{A B}+\theta_{\text {offset }}^{B P}+c+\epsilon_{1} \\
& T_{i}^{A P}=T_{i}^{A B}+\theta_{\text {offset }}^{B P}+\theta_{\text {skew }}^{B P} \cdot\left(T_{i}^{A B}-T_{1}^{A B}\right) \\
& +c+\epsilon_{i}, 1<i \leq N
\end{aligned}
$$

where $\theta_{\text {offset }}^{B P}$ and $\theta_{\text {skew }}^{B P}$ denote the clock offset and skew between $B$ and $P$. The parameters $c$ and $\epsilon_{i}$ stand for the fixed portion and random portion of packet delay $[2,7,8]$. If the set of time stamp differences is denoted by $T=$ $\left[T_{1}^{A P}-T_{1}^{A B} \cdots T_{N}^{A P}-T_{N}^{A B}\right]^{\mathrm{T}}$, then it can be expressed as follows:

$$
T=H \Theta+C+E
$$

where $\Theta=\left[\theta_{\text {offset }}^{B P} \theta_{\text {skew }}^{B P}\right]^{\mathrm{T}}, C=[c \cdots c]^{\mathrm{T}}, E=\left[\epsilon_{1} \cdots \epsilon_{N}\right]^{\mathrm{T}}$, and $H=\left[\begin{array}{cccc}1 & 1 & \cdots & 1 \\ 0 & T_{2}^{A B}-T_{1}^{A B} & \cdots & T_{N}^{A B}-T_{1}^{A B}\end{array}\right]^{\mathrm{T}}$.

Consequently, using the Equation $3, B$ can be synchronized with $P$. In addition, Equations 1 to 3 can also be used to synchronize the reference node by substituting $A$ for $B$. In order to improve the precision, TTS uses $N+2$ broadcast packets to provide $N$ sync-points and the node records time stamp at MAC layer to remove send, receive, and access time. It is worth mentioning that $B$ utilizes reference broadcast scheme to eliminate the transmitter-side non-determinism, thus $\epsilon_{i}^{B P} \approx \epsilon_{i}^{A P} / 2$.

\section{The multi-hop algorithm of TTS}

In practical WSN, a common node always gets information from the root node through a multi-hop path. Thus, the single-hop TTS is not enough to achieve the whole network synchronization. In these scenarios, the multihop TTS should be used to synchronize nodes beyond the communication range of the root node.

We utilize a simple demo illustration to show the difference between common protocol and multi-hop TTS. In Figure 3, suppose that only root node 1 has the global time. For common protocols, first, the root node synchronizes the four nodes connected with it. Then, nodes 3 and 4 are selected to synchronize nodes of level 3 . However, TTS only selects two pairs of nodes to synchronize all common nodes. For example, nodes 3 and 1 perform pair-wise message exchange to synchronize nodes $2,3,4,6,7,8$, and 9 because they all locate within the broadcast range of node 3. Similarly, nodes 4 and 1 exchange packets to synchronize nodes $2,3,4,5,8,9,10$, and 11 . Therefore, the TTS aims at finding all the synchronization pairs from the whole network.

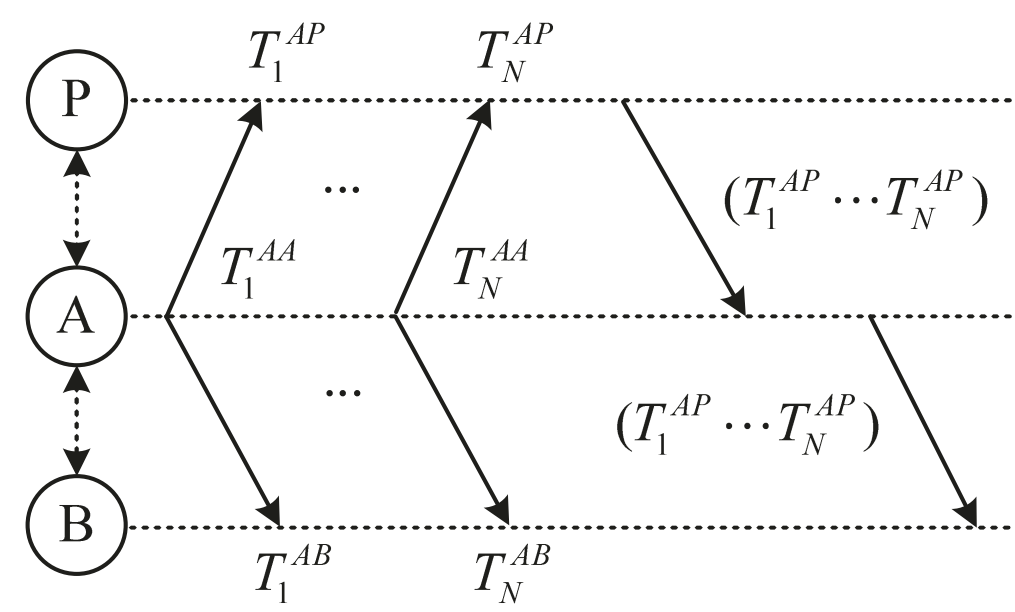

Figure 2 Clock synchronization model of TTS. 


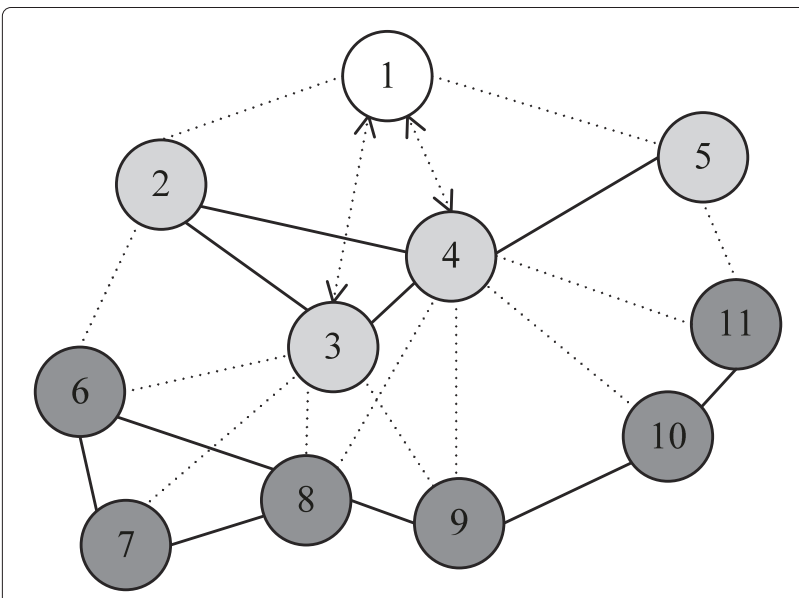

Figure 3 Network-wide TTS synchronization for a simple network.

\subsection{Synchronization problem formulation}

The procedure of searching synchronization pairs can be divided into some sub-problems. Suppose that reference nodes of level $2 i-2$ have been found whereas reference nodes of level $2 i$ are not found. This means that nodes of level $2 i$ and $2 i+1$ are uncovered. We utilize an undirected graph $G(V, E)$ to model the topology of nodes of level $2 i$ and $2 i+1$. Let $V=V_{E} \cup V_{O}$ represent the set of nodes. $V_{E}$ is the set of level $2 i$ nodes and $V_{O}$ is the set of level $2 i+$ 1 nodes. Edge set $E$ stands for the set of communication link and node self-loop. The link $(v, e) \in E$ if it satisfies: at least one of the two elements belongs to $V_{E}, v$ could communicate with $e$ or $v=e$. Thus, the edges connecting nodes in $V_{O}$ only are not included. Then, the sub-problem can be described as finding minimum number of reference nodes from $V_{E}$ to cover all nodes in $V$.

Theorem 1. The process of the sub-problem is NPcomplete.

Claim 1. The sub-problem is in NP.

Proof. The following verifier for the sub-problem runs in polynomial time.

For $\langle(V, E, K), R\rangle$

If all the followings are all true then accept else reject:

$R$ is a subset of $V_{E}$

$|R| \leq K$

$\forall v \in V \exists e \in R[(v, e) \in E]$.

Claim 2. Set covering problem $(\mathrm{SCP}) \leq p$ sub-problem

Proof. Suppose a function with the input $(V, S, K)$, which is a SCP instance, and output $(V, E, K)$ which stands for the sub-problem. Let $S=\left\{S_{e}: e \in V_{E}\right\}$ and $S_{e}=\{v \in V:(v, e) \in E\}$. There are $|V|$ vertices and $\sum_{e=1}^{|S|}\left|S_{e}\right|$ edges. We now show that the function is a polynomial time reduction of SCP to sub-problem.

We assume that $J$ is a $S$-cover of $V$ and let $R=\left\{e \in V_{E}\right.$ : $\left.S_{e} \subset J\right\}$. Suppose $|J|=K$, then $|R|=K$. We claim that $R$ could cover all elements of $V$. Suppose $v$ is a common node of $V$. So we have $\left\{v \in S_{e}: S_{e} \subset J\right\}$. According to the definition of $S_{e},(v, e) \in E$. The definition of $R$ shows that $e \in R$. Thus, $v$ could be covered by an elemente of $R$.

Then, we suppose $R$ with size of $K$ could cover all the elements of $V$. let $J=\left\{S_{e}: e \in R\right\}$, then $|J|=K$. We will show that $J$ is a S-cover of $V$. For a common node $v \in V$, we know that $\exists e \in R[(v, e) \in E]$. Thus, according to the definition of $S_{e}, v \in S_{e}$. The definition of $J$ shows that $S_{e} \in$ $J$. We can see that $v \in J$ and $J$ is the cover of $V$.

\subsection{Multi-hop process of TTS}

There is no efficient way to solve the SCP. A famous sub-optimal solution for $\mathrm{SCP}$ is the greedy algorithm. We propose the multi-hop TTS, which is a distributed greedy algorithm, to solve the sub-problem. This algorithm only uses even layer node as reference node, so it is also named as even layer TTS.

Every node in the network is assigned a unique ID to identify the source of message. A node obtains its level through the level discovery phase [7]. The parameter $\operatorname{syn}_{v}$ represents the cover state of node $v$, that is, $\operatorname{syn}_{v}=1$ when $v$ itself is the reference node or $v$ is located within the broadcast domain of a reference node, and $\operatorname{syn}_{v}=0$ otherwise. Let num $v$ denote the number of $v$ 's neighbors with parameter syn $=0$. The variable num can also be obtained initially from the level discovery phase. The distributed algorithm can be described as:

1. Nodes in $V_{E}$ are divided into two types, reference nodes and common nodes. According to CSMA, common node $e \in V_{E}$ waits for some random time to avoid collision and to ensure that the wireless channel is free. Then, e broadcasts a packet containing $\operatorname{num}_{e}$ and its own identity. Meanwhile, $e$ also collects corresponding variables from the neighbors. Having received all such variables, $e$ computes out and broadcasts the maximum value $\left\{\max _{e}=\max \left(\operatorname{num}_{v}\right), v \in V_{E},(v, e) \in E\right\}$ to all the neighbors. In this step, the number of broadcasted messages is less than $2\left|V_{E}\right|$.

2. Common node $e \in V_{E}$ judges whether it needs to be a reference node or remains a common node. Node $e$ becomes a candidate of the reference node if num is the largest variable among all the two-hop neighbors of $e$. That is,

$$
\operatorname{num}_{e}=\max \left(\max _{v}\right), \forall v \in V_{E},(v, e) \in E
$$


If candidate $e$ timeouts after some random time without receiving any reference message (refmsg), it becomes a reference node and broadcasts refmsg immediately. Otherwise, node e is still a common node.

Here, we present a simple example of this step. In Figure 3, suppose that all nodes of levels 2 and 3 are uncovered, that is, their parameters syn are all equal to zero. Now the sub-problem is to find all the reference nodes of level 2. First, every node of level 2 exchanges num with the neighbors of the same level. From Figure 3, we can see that num $2=|\{2,3,4,6\}|=$ 4, num $_{3}=|\{2,3,4,6,7,8,9\}|=7$, num $_{4}=\mid\{2,3,4,5$, $8,9,10,11\} \mid=8$ num $_{5}=|\{4,5,11\}|=3$. Then, node 4 becomes a reference node because num 4 is the largest among all the neighbors. The iterative process continues until nodes 6 and 7 are also covered.

3. If node $v \in V$ with parameter $\operatorname{syn}_{v}=0$ becomes a reference node or has received refmsg, it sets the parameter $\operatorname{syn}_{v}=1$ and broadcasts a cover state message (covstate). This broadcasting process also adopts CSMA scheme.

4. Each common node $e \in V_{E}$ updates num $e$ after receiving all covstates.

$$
\operatorname{num}_{e}=\sum\left(1-\operatorname{syn}_{v}\right), \forall v \in V,(v, e) \in E .
$$

Steps 1 to 4 are repeated until all nodes in $V$ are lying within the broadcast range of the reference nodes. Then, each reference node randomly selects a level $2 i-$ 1 neighbor to make a synchronization pair, and this sub-problem is finished. When all these sub-problems have been accomplished, the network-wide synchronization will be performed along the synchronization pairs.

\subsection{The performance of multi-hop TTS}

Theorem 2. The multi-hop TTS yields a set of size at most $\ln \Delta+2$ times the size of the optimal set.

\section{Proof. See Appendix.}

In multi-hop TTS, more than one reference node is found in one iteration process and only a small number of nodes become reference nodes. Hence, the actual number of iterations is far less than $\left|V_{E}\right|$ and the maximum complexity of step 1 is $O\left(\left|V_{E}\right|^{2}\right)$. In steps 2 and 3 , every node broadcasts the message covstate and some nodes broadcast the message refmsg, so the complexity is $O(|V|)$. Therefore, the total complexity of the whole process is $\left\{O\left(\left|V_{E}\right|^{2}\right)+O(|V|)\right\}$. Note that the communication messages needed to determine the synchronization pairs are one-off.
The TTS reduces the synchronization messages significantly because it makes full use of node's broadcast characteristic. A node's broadcast range can be divided into three parts: area that covers neighbors in the previous layer, area that covers neighbors in the same layer, and area that covers neighbors in the next layer. In common protocols, a synchronized node can only synchronize its neighbors in the next layer. However, the reference node of TTS is able to synchronize all neighbors in the same and next layer. Therefore, the broadcast domain utilization of TTS is higher than that of common protocols. As depicted in Figure 1a,b, TTS gives a roughly $2 \times$ larger maximum synchronization area compared with other protocols. Moreover, the distributed TTS chooses the most appropriate nodes as the reference nodes. By minimizing reference nodes, TTS significantly reduces the number of timing packets which are heavy overheads in terms of energy consumption.

The TTS halves the synchronization hops and effectively reduces the multi-hop synchronization error by synchronizing nodes of two adjacent levels. In Figure 1a,b, suppose that both $P$ and $P^{\prime}$ have been synchronized and the number of hops from a common node to the root node is $H$. The goal of traditional protocols is to ensure the shortest path, which means the least number of hops, to the root node. They use $P^{\prime}$ of level $i^{\prime}$ to synchronize the nodes of level $i^{\prime}+1$. Thus, the number of synchronization hops of traditional protocols is $H$. However, TTS selects $P$ of level $2 i-1$ and $A$ of level $2 i$ as a synchronization pair to synchronize level $2 i$ and $2 i+1$ nodes. The number of synchronization hops of TTS can be expressed as [ $(H+1) / 2]$, which means the floor of $(H+1) / 2$, i.e., the largest integer less than or equal to $(H+1) / 2$. Since the synchronization error increases with the number of synchronization hops, the accuracy of TTS is higher compared with previously known protocols.

Fewer synchronization hops and less overhead mean lower convergence time that is needed to achieve network-wide synchronization. Let $C$ be the channel capacity, $\rho$ be the node density, and $r$ be the node broadcast radius. Then, the node throughput equals $k C /\left(\pi r^{2} \rho\right)$ where $k$ is the channel utilization. Traditional protocols exchange $N$ timing packets to accomplish synchronization, and reference [2] shows that the most suitable value of $N$ is 30 . The convergence time of traditional protocols can be expressed as:

$$
C T_{t}=\frac{\pi r^{2} P}{k C} \rho_{t} N H_{\max }
$$

where $H_{\max }$ is the maximum number of hops of a network and $P$ is the size of synchronization packet. Our even layer TTS needs $N+2$ messages, and its synchronization hops 


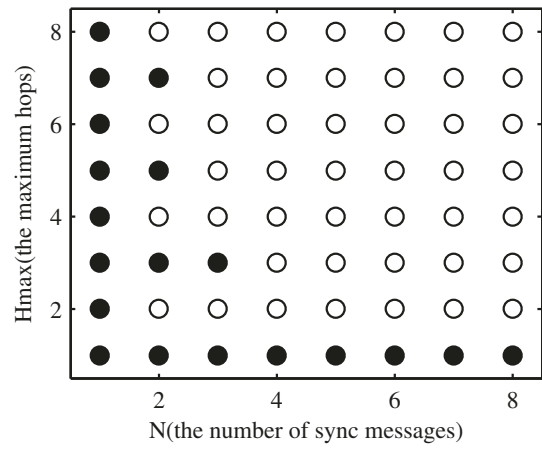

(a)

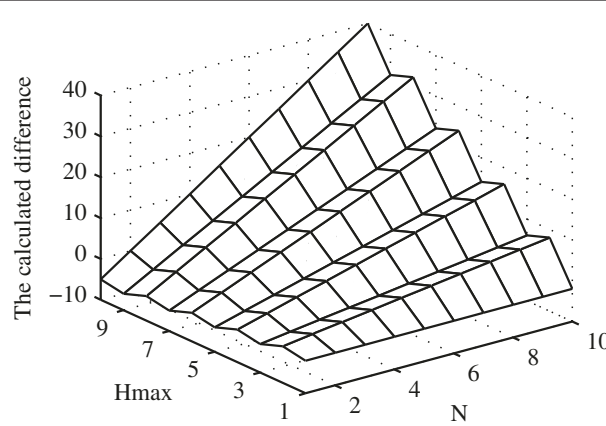

(b)

Figure 4 The comparison and difference between $\boldsymbol{N} \boldsymbol{H}_{\max }$ and $(\boldsymbol{N}+\mathbf{2})\left[\left(\boldsymbol{H}_{\max }+\mathbf{1}\right) / \mathbf{2}\right]$. (a) The comparison of $N H_{\max }$ and $(N+2)\left[\left(H_{\max }+1\right) / 2\right]$. The white dot represents that the former is larger than the latter and the black dot conversely. (b) The difference between $\mathrm{NH}_{\max }$ and $(N+2)\left[\left(H_{\max }+1\right) / 2\right]$

are half of that of common protocols. The convergence time of TTS is given by:

$$
\mathrm{CT}_{e \mathrm{TTS}}=\frac{\pi r^{2} P}{k C} \rho_{e \mathrm{TTS}}(N+2)\left[\frac{H_{\mathrm{max}}+1}{2}\right] .
$$

Equations 6 and 7 show that the convergence time raises with the increasing of the value of $\rho$. As shown earlier, TTS requires less synchronization nodes to synchronize the whole network and thus $\rho_{e T T S}<\rho_{t}$. So TTS has less convergence time when just considering about the parameter $\rho$. In addition, the parameter $k$ decreases with the growth of $\rho$ in basic CSMA which is a very important MAC layer protocol $[16,17]$. Then, we assume $\rho_{t}=$ $\rho_{e \text { TTS }}$ and compare $N H_{\max }$ with $(N+2)\left[\left(H_{\max }+1\right) / 2\right]$. We turn to MATLAB to verify TTS has less convergence time. In Figure $4 \mathrm{a}$, the white dot denotes that $\mathrm{NH}_{\max }$ is relative large. When the number of $N$ is larger than 3 , the TTS always has less convergence time compared with common protocols in multi-hop networks. Actually, in order to calculate the clock skew, $N$ is usually very large. Figure $4 \mathrm{~b}$ plots the calculated difference between $N H_{\max }$

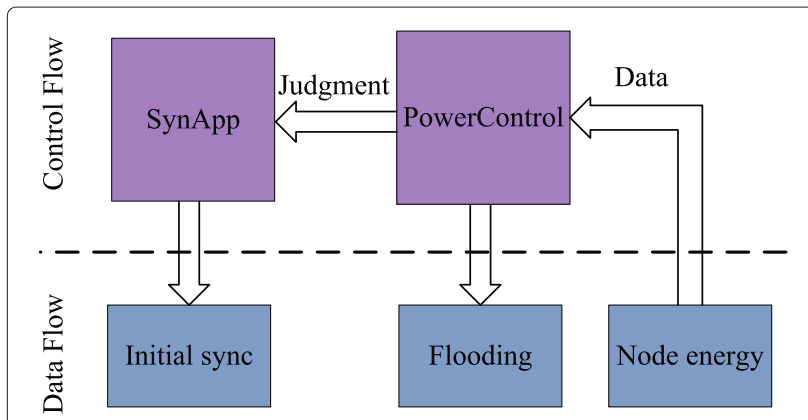

Figure 5 Example of the power control scheme as an application service. and $(N+2)\left[\left(H_{\max }+1\right) / 2\right]$. The figure shows that the difference increases with the growths of both $N$ and $H_{\max }$. Therefore, TTS performs better in networks with large maximum number of hops.

\subsection{Power control scheme}

In WSN, nodes have to work as energy-efficient as possible due to limited energy supply. In the protocol proposed above, only even layer nodes are chosen as reference nodes. Those selected nodes consume more power compared with odd layer nodes. Here, we present odd layer TTS to solve this problem.

The root node broadcasts $N$ packets containing the sender's time stamps which denote the global time. Each level 2 node obtains the corresponding local time at message reception. The node calculates the clock offset by working out the difference between the global and local time and then becomes synchronized. Nodes of

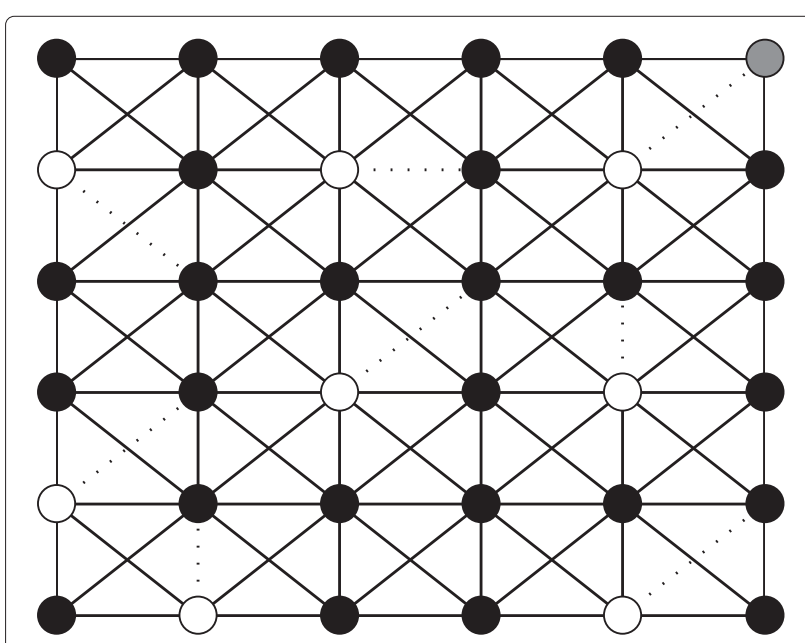

Figure 6 The selected synchronization pairs in $6 * 6$ network. 


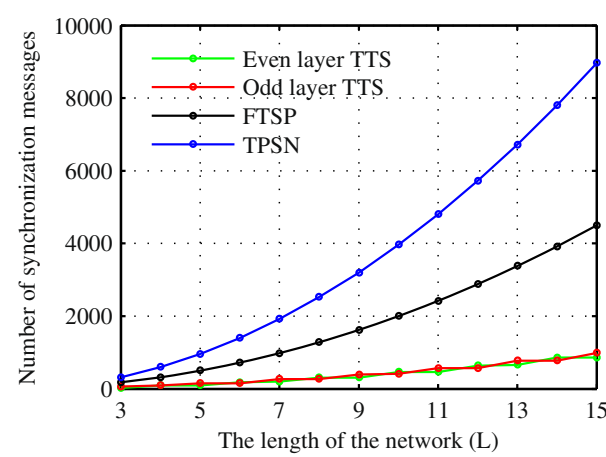

(a)

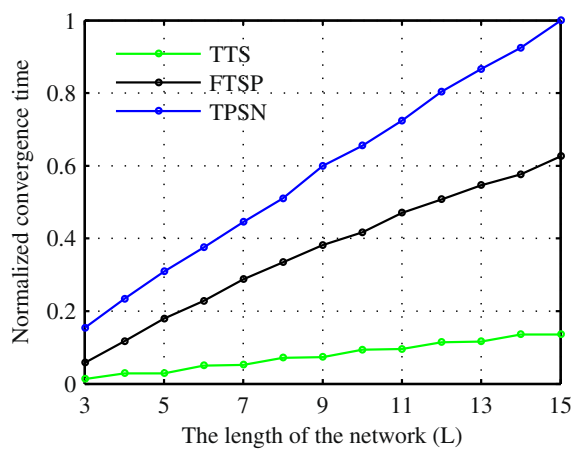

(b)

Figure 7 Message overhead and convergence time of different protocols. (a) The message overhead differences between different protocols. (b) The convergence time differences between different protocols.

level 2 are synchronized by S-RS which has lower accuracy compared with R-RS. After that, odd level nodes use the above distributed algorithm to achieve networkwide synchronization. Note that even layer TTS and odd layer TTS can be performed alternately in practical WSN protocols.

Figure 5 shows a possible way of achieving power control by utilizing two types of TTS. Each synchronization node with less power remaining sends a warning message containing its own identity and level to the root node. An application PowerControl is generated by the root node to collect the energy consumption state of the whole network. PowerControl analyzes the data and changes the synchronization mode when the powers of most current reference nodes are too low. Then, PowerControl starts a flooding process to notify all the nodes. Depending on the judgment of PowerControl, SynAPP initials the whole network synchronization.

\section{Experiment results}

5.1 Message overhead and convergence time comparison The experiment scenario is a $L * L$ network with grid topology, in which each node communicates with the

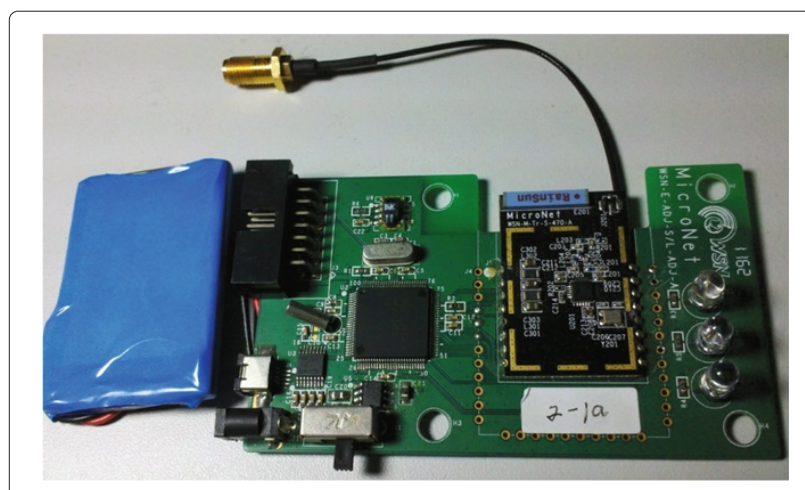

Figure 8 Close-up of the common sensor node. neighbors only. An example of the topology connection state is shown in Figure 6. The gray dot deployed in the upper right corner is the root node. The white dot denotes the reference node which connects with its parent node via dashed line. The reference node and parent node makes a synchronization pair, and all synchronization pairs are selected by even layer TTS. In the figure, only the most appropriate even layer nodes are chosen as reference nodes, and all nodes could be located within the broadcast domain of the synchronization pairs. The next experiment focuses on the overhead and convergence time required by different protocols.

We evaluate the message overhead required by TTS, TPSN, and FTSP. In TPSN, each node except the root node forms a synchronization pair with its parent and each synchronization pair demands $2 N$ timing messages. Therefore, TPSN needs $2 N\left(L^{2}-1\right)$ packets to achieve synchronization of the network mentioned before. If FTSP is adopted to synchronize the network, every node sends its time stamp to other nodes, so the number of required packets is $N L^{2}$. Notice that RBS demands $O\left(L^{4}\right)$ packets in a single-hop network, which is too large compared with both TPSN and FTSP. The value of $N$ is set to be 20. It can be seen from Figure 7a that TTS requires a much lower number of overheads compared with both TPSN and FTSP. The overhead gaps between TTS and other protocols become greater as $L$ is increasing. This indicates that TTS performs better with regard to energy consumption versus TPSN and FTSP in large-scale networks. Furthermore, the decrease of packets also means the reduction of network conflict and global time error.

Another experiment is carried out to show the convergence time differences between TTS and other classical protocols. Although RBS is one of the most important protocols, it does not have a clear multi-hop synchronization scheme. Also, there is no root node in RBS, so the common nodes can only get relative time. Therefore, we 


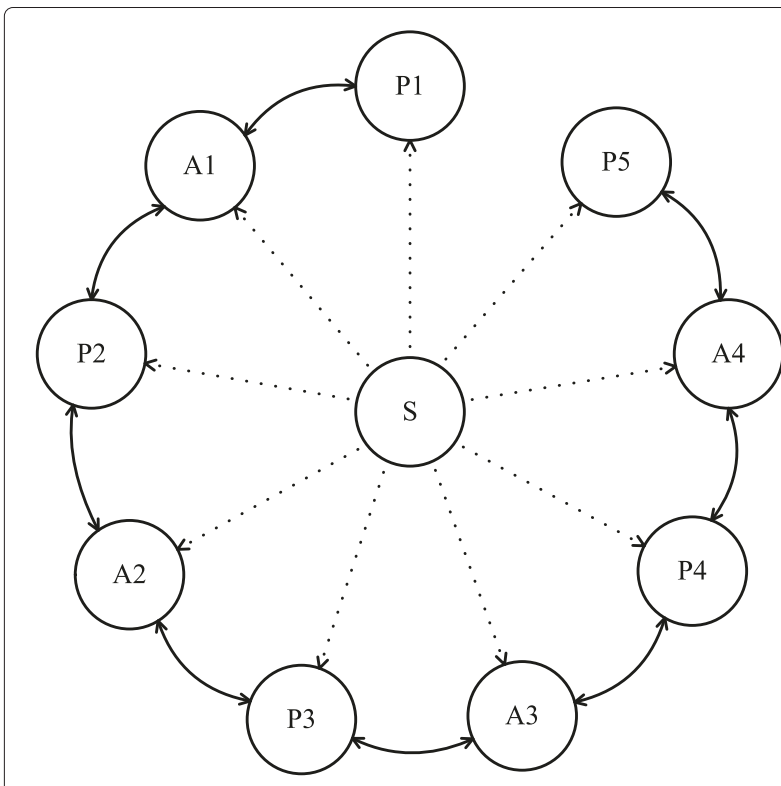

Figure 9 Wireless sensor network communication topology of ten sensor nodes.

just simulated TPSN, FTSP, and TTS on MATLAB. In the simulation, the TPSN first established a tree hierarchy and each node synchronized with its parent through pair-wise message exchange. The FTSP achieved networkwide synchronization by flooding. In all the three protocols, common nodes communicated with their neighbors through CSMA protocol. Figure 7b shows the convergence times of different protocols. Each point represents the average of 100 experiment results. The convergence times of the three protocols all increase with the network size because the number of nodes and maximum hops increase with the network scale. The convergence time of TTS is always less than the other two protocols and the gaps raise with $L$. This is consistent with Equations 6 and 7. The reason lies in two aspects: TTS has less message overhead; TTS halves the synchronization hops. Therefore, TTS converges fast and performs significantly better in large-scale WSN.

\subsection{The synchronization accuracy of TTS}

We use the implementation of TTS on common sensors to carry out the advantages of TTS described above. The hardware of the sensor consists of processor board, radio, and battery. We base our design on the microcontroller TI MSP430F5438 and on the radio chip TI CC1100E. The processor adopts $16 \mathrm{MHz}$ external crystal oscillator, so its timer maintains a local clock with the resolution of about $0.0625 \mu \mathrm{s}$. The central frequency of the radio chip is 470 $\mathrm{MHz}$, and its data rate is set to be $100 \mathrm{kBaud}$. We utilize the 4.5-V lithium rechargeable battery as the power of the sensor. The sensor also provides lots of external interfaces (e.g., RS232 serial port, JTAG emulator interface, LED lights). Figure 8 shows a prototype of this hardware design.

Figure 9 shows the experiment scenario and the network connection state. The experiment involves a super node and nine common nodes. The topology of the ten nodes is enforced in software in order to facilitate the realization. The topology structure shows that all common nodes could be covered by the broadcast domain of the super node $S$. $S$ queries and collects the clock time from $P_{i}$ once per $7.9 \mathrm{~s}$. It sends the time readings to PC through 115,200 -baud serial link. Then, $S$ broadcasts a start message, so that all nodes begin to re-synchronize. The synchronization process is started by the root node $P_{1}$ which maintains the global time. Other common nodes communicate with the neighbors and exchange synchronization messages according to the adopted protocols demonstrated below.

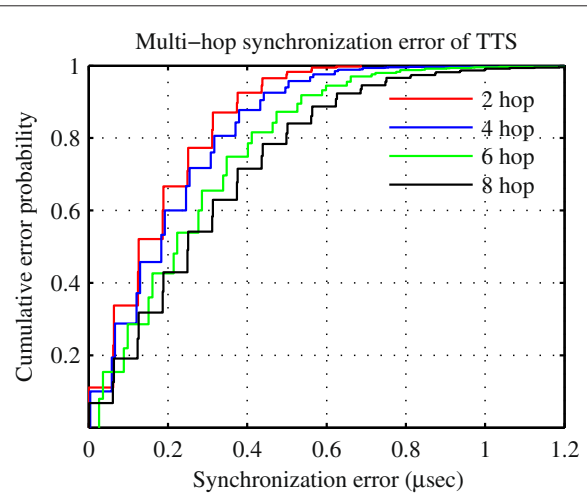

(a)

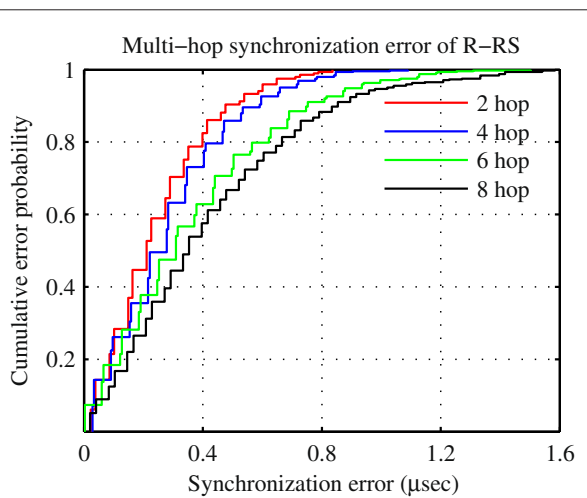

(b)

Figure 10 The multi-hop synchronization errors of TTS and R-RS. (a) The multi-hop synchronization error of TTS. (b) The multi-hop synchronization error of R-RS. 
Table 1 Statistics of synchronization variances over multi-hop

\begin{tabular}{lcccc}
\hline Sensor & \multicolumn{1}{c}{$\boldsymbol{P}_{\mathbf{2}}$ (two hops) } & $\boldsymbol{P}_{\mathbf{3}}$ (four hops) & $\boldsymbol{P}_{\mathbf{4}}$ (six hops) & $\boldsymbol{P}_{\mathbf{5}}$ (eight hops) \\
\hline TTS variance & 11.83 & 19.13 & 29.37 & 39.84 \\
R-RS variance & 23.52 & 31.08 & 53.77 & 70.80 \\
\hline
\end{tabular}

- TTS: $P_{i}$ and $A_{i}$ exchange messages, and $P_{i+1}$ synchronizes with $P_{i}$ by overhearing the broadcasts from $A_{i}$. After that, $P_{i+1}$ starts to exchange messages with $A_{i+1}$. This process continues until $P_{5}$ is synchronized.

- R-RS: At first, $S$ broadcasts a reference message. $P_{1}$ and $A_{1}$ records the reception time; then, $P_{1}$ sends the recorded time to $A_{1}$. $A_{1}$ synchronizes with $P_{1}$ by computing out the time difference. This process continues until $P_{5}$ is also synchronized with $A_{4}$.

Each scenario consists of 500 trials. We compute out the synchronization error between $P_{1}$ and $P_{i}$. The distributions of clock error are shown in Figure 10, and the variances are summarized in Table 1. As shown in Figure 10a,b, the synchronization error increases with hop distance. This means that, both in TTS and R-RS, nodes with lower hops perform better than those with higher hops. The thresholds at confidence of $90 \%$ in two, four, six, and eight hops of TTS are about $0.36,0.43,0.52$, and 0.60 $\mu \mathrm{s}$, respectively. The values of TTS are better than that of the R-RS $(0.48,0.59,0.75$, and $0.84 \mu \mathrm{s})$. From Table 1 , the four-hop variance of TTS is smaller than the two-hop variance of R-RS. Although the eight-hop variance of TTS is larger than the four-hop variance of R-RS, they are in the same order of magnitude and the former is smaller than the six-hop variance of R-RS. In summary, TTS dramatically reduces the variance of global synchronization error. The reason is that TTS halves the synchronization hops.

\section{Conclusions}

In this paper, we have introduced the two-hop time synchronization for sensor networks. We show that TTS requires less message exchanges to achieve network-wide synchronization. The efficacy of this claim is verified via simulations on MATLAB. The convergence time of TTS is also very low. We argue that TTS is more accurate in largescale networks because it halves synchronization hops. We verify this claim by implementing TTS and R-RS on common sensors. The results show that TTS performs better than R-RS in multi-hop synchronization.

The single-hop TTS can be used as the substitute of R-RS. In TTS, the reference node records the time stamps when broadcasting reference messages. The reference node and its children nodes can be synchronized with a selected parent node. We utilize a distributed algorithm to extend the single-hop TTS to multi-hop synchronization. The algorithm minimizes the number of reference nodes and halves the synchronization hops compared with other protocols. Thus TTS is a distributed, scalable, energyefficient, and accurate solution to the problem of sensor network clock synchronization.

Our future work will focus on several aspects. First, TTS is sensitive to the dynamic topology change, so we would like to make it more robust. Then, we plan to verify the performance of TTS in a real-world application. The most attractive scenarios are those with great range of hardware platforms, time-varying network topology, and multi-user applications.

\section{Appendix}

Theorem 3. The multi-hop TTS yields a set of size at most $\ln \Delta+2$ times the size of the optimal set.

Proof. Suppose that OPT $\subset V_{E}$ is the optimal set. A node in $V$ either belongs to OPT or has a neighbor in OPT. The set of nodes covered by $i^{*} \in$ OPT is called $S_{i^{*}}$. If a node is covered by more than one neighbors in OPT, we arbitrary assign it to one of such sets.

We use the charging scheme to prove the efficacy of TTS. The total cost of an optimal set $S_{i *}$ is 1 and each node of the set is charged $1 / \mid S_{i^{*}}$. In the following, we prove that the total cost of TTS is at most $\ln \Delta+2$ for each set $S_{i^{*}}$.

Each time we select a reference node, we charge the new nodes that become covered in this step. A node is charged only once because it gets covered only once. Suppose the number of nodes covered by a new reference node $i$ is $N_{i}$. Then, if node $v$ in the set of $S_{i^{*}}$ is covered by $i$, it gets charged $1 / N_{i}$. According to step 1 of multi-hop TTS, $N_{i}$ is the largest among all the two-hop neighbors and therefore $N_{i}>N_{i^{*}}$. So node $v$ gets charged at most $1 / N_{i^{*}}$. Let $d_{i^{*}}$ represent the number of neighbors of node $i^{*}$. Therefore, the first node in the set of $S_{i^{*}}$ gets charged at most $1 /\left(d_{i^{*}}+1\right)$ and the $j$ th node gets charged at most $1 /\left(d_{i^{*}}-j+2\right)$. Adding up all the charges of nodes in $S_{i^{*}}$, we get

$$
\frac{1}{d_{i^{*}}+1}+\frac{1}{d_{i^{*}}}+\cdots+\frac{1}{2}+1<\ln \Delta+2
$$

where $\Delta$ is the maximum degree of $V$.

\section{Abbreviations}

ATS: Average TimeSynch; CAS: Chinese Academy of Sciences; DMTS: Delay measurement time synchronization; FTSP: Flooding time synchronization protocol; KFMP: Vector Kalman filter using multiple parents; LTS: Lightweight time synchronization; MAC: Media access control; PBS: Pair-wise broadcast synchronization; P-WS: Pair-wise synchronization; RBS: Reference broadcast synchronization; R-RS: Receiver-receiver synchronization; SCP: Set covering problem; S-RS: Sender-receiver synchronization; SSTC: Shanghai Science and Technology Commission; TPSN: Timing-sync protocol for sensor networks; TTS: Two-hop time synchronization; WSN: Wireless sensor networks. 


\section{Competing interests}

The authors declare that they have no competing interests.

\section{Acknowledgements}

This work is supported in part by the 'Strategic Priority Research Program' of the Chinese Academy of Sciences (CAS) under Grant No. XDA06020301 and Shanghai Science and Technology Commission (SSTC) research projects under Grant No. 12DZ2293200

\section{Received: 1 July 2013 Accepted: 7 March 2014}

Published: 12 March 2014

\section{References}

1. F Wang, P Zeng, $\mathrm{H}$ Yu, Y Xiao, Random time source protocol in wireless sensor networks and synchronization in industrial environments. Wireless Commun. Mobile Comput. 13(8), 798-808 (2013)

2. J Elson, L Girod, D Estrin, Fine-grained network time synchronization using reference broadcasts. SIGOPS Oper. Syst. Rev. 36(SI), 147-163 (2002)

3. S PalChaudhuri, AK Saha, DB Johnson, Adaptive clock synchronization in sensor networks, in Proceedings of the 3rd international symposium on Information processing in sensor networks (ACM IPSN '04, New York, NY, USA, 2004), pp. 340-348

4. A Marco, R Casas, J Ramos, V Coarasa, A Asensio, M Obaidat, Synchronization of multihop wireless sensor networks at the application layer. Wireless Commun. IEEE. 18, 82-88 (2011)

5. J van Greunen, J Rabaey, Lightweight time synchronization for sensor networks, in Proceedings of the 2nd ACM international conference on Wireless sensor networks and applications (ACM WSNA '03, New York, NY, USA, 2003), pp. 11-19

6. ML Sichitiu, C Veerarittiphan, Simple, accurate time synchronization for wireless sensor networks, in Wireless Communications and Networking Conference, vol. 2 (IEEE WCNC '03, New York, NY, USA, 2003), pp. 1266-1273 vol. 2

7. S Ganeriwal, R Kumar, MB Srivastava, Timing-sync protocol for sensor networks, in Proceedings of the 1st international conference on Embedded networked sensor systems (ACM SenSys '03, New York, NY, USA, 2003), pp. 138-149

8. M Maróti, B Kusy, G Simon, A Lédeczi, The flooding time synchronization protocol, in Proceedings of the 2nd international conference on Embedded networked sensor systems (ACM SenSys '04, New York, NY, USA, 2004), pp. 39-49

9. S Ping, Delay measurement time synchronization for wireless sensor networks. (Intel Research Berkeley Lab, IRB-TR-03-013, CA, USA, 2003)

10. Klae Noh, E Serpedin, K Qaraqe, A new approach for time synchronization in wireless sensor networks: Pairwise broadcast synchronization. Wireless Commun. IEEE Trans. 7(9), 3318-3322 (2008)

11. Y Zeng, B Hu, S Liu, Vector Kalman filter using multiple parents for time synchronization in multi-hop sensor networks, in 5th Annual IEEE Communications Society Conference on Sensor, Mesh and Ad Hoc Communications and Networks (Inst. of Elec. and Elec. Eng. Computer Society Secon'08, NJ, USA, pp. 413-421

12. L Zheng, W Ge, H Qiu, Master synchronization in physical-laye communications of wireless sensor networks. EURASIP J. Wirel. Commun. Netw. 2010, 108:1-108:9 (2010)

13. L Schenato, F Fiorentin, Average TimeSynch: A consensus-based protocol for clock synchronization in wireless sensor networks. Automatica. 47(9), 1878-1886 (2011)

14. KY Cheng, KS Lui, YC Wu, V Tam, A distributed multihop time synchronization protocol for wireless sensor networks using Pairwise Broadcast Synchronization. Wireless Commun. IEEE Trans. 8(4), 1764-1772 (2009)

15. KL Noh, YC Wu, K Qaraqe, B Suter, Extension of pairwise broadcast clock synchronization for Multicluster sensor networks. EURASIP J. Adv. Signal Process. 2008, 286168 (2008)
16. G Bianchi, L Fratta, M Oliveri, Performance evaluation and enhancement of the CSMA/CA MAC protocol for 802.11 wireless LANs IEEE, PIMRC '96, NY, USA, 1996), pp. 392-396 vol. 2

17. E Ziouva, T Antonakopoulos, CSMA/CA performance under high traffic conditions: throughput and delay analysis. Comput. Commun. 25(3), 313-321 (2002)

doi:10.1186/1687-1499-2014-39

Cite this article as: Wang et al:: Two-hop time synchronization protocol for sensor networks. EURASIP Journal on Wireless Communications and Networking 2014 2014:39.

\section{Submit your manuscript to a SpringerOpen ${ }^{\mathcal{O}}$ journal and benefit from:}

- Convenient online submission

- Rigorous peer review

- Immediate publication on acceptance

- Open access: articles freely available online

- High visibility within the field

- Retaining the copyright to your article

Submit your next manuscript at $>$ springeropen.com 